\title{
Impaired death-associated protein kinase-mediated survival signals in 5-fluorouracil-resistant human endometrial adenocarcinoma cells
}

\author{
TETSUJI TANAKA ${ }^{1^{*}}$, TAO BAI $^{2 *}$, SAORI TOUJIMA ${ }^{3}$, TOMOKO UTSUNOMIYA $^{3}$, \\ HIROTOSHI UTSUNOMIYA ${ }^{4}$, KAZUNORI YUKAWA ${ }^{5}$ and JUNKO TANAKA ${ }^{6}$
}

${ }^{1}$ Santamaria Hospital, 13-15 Shinjo-cho, Ibaraki, Osaka 567-0884, Japan; ${ }^{2}$ Department of Neurology,
The University of Chicago, Maryland Ave., Chicago, IL 60637, USA; Departments of ${ }^{3}$ Obstetrics and
Gynecology and ${ }^{4}$ Pathology, Wakayama Medical University, 811-1 Kimi-idera, Wakayama 641-0012;
${ }^{5}$ Department of Physiology, Faculty of Pharmacy, Meijo University, 150 Yagotoyama,
Tempaku-ku, Nagoya 468-8503; ${ }^{6}$ Graduate School of Intercultural Studies,
Kobe University, 1-2-1 Tsurukabuto, Nada-ku, Kobe 657-8501, Japan

Received January 5, 2012; Accepted February 15, 2012

DOI: $10.3892 / o r .2012 .1774$

\begin{abstract}
A recent study showed that both 5-fluorouracil (5FU)-stimulated apoptosis and Fas-mediated apoptosis in human endometrial adenocarcinoma cells are enhanced by targeted knockdown of endogenous death-associated protein kinase (DAPK) with DAPK small-interfering RNAs. Therefore, we investigated the DAPK survival signals in three 5FU-resistant subclones. DAPK knockdown did not enhance 5FU-stimulated or Fas-mediated apoptosis in any of the three 5FU-resistant subclones, but the subclones acquired resistance to VP16-stimulated cell death that was DAPK-independent. Semiquantitative flow cytometric analyses showed that there was no differential expression in nine cell surface antigens, including Fas, and six intracellular molecules, including DAPK, that may regulate cell death or survival between the parent cells and 5FU-resistant cells. DAPK mRNA and protein were expressed in the 5FU-resistant subclones at similar levels to the parent cells. These results indicate that acquisition of 5FU-resistance may be accompanied by impairment of common apoptotic signals regulating both DAPK-dependent and DAPK-independent pathways.
\end{abstract}

\section{Introduction}

The death-associated protein kinase (DAPK) cDNA was isolated from human cervical carcinoma cells as a positive mediator of apoptosis triggered by interferon- $\gamma(1)$. Recent investigations have

Correspondence to: Dr Tetsuji Tanaka, Santamaria Hospital, 13-15 Shinjo-cho, Ibaraki, Osaka 567-0884, Japan

E-mail: tetanaka@santamaria-med.jp

*Contributed equally

Key words: death-associated protein kinase, 5-fluorouracil, drugresistance, apoptosis revealed that DAPK functions as a $\mathrm{Ca}^{2+} /$ calmodulin-dependent serine/threonine kinase to regulate cell death or survival (1-16). However, the physiological functions of DAPK have not been fully clarified. Loss of DAPK expression has been implicated in tumorigenesis and metastasis $(3,4,13)$, suggesting a crucial role for DAPK in the apoptotic process under pathological conditions. On the other hand, inhibition of DAPK expression in HeLa cells, 3T3 fibroblasts, primary human vascular smooth muscle cells and various human uterine cancer cells using an antisense DAPK or small-interfering RNAs (siRNAs) for DAPK was found to increase apoptosis $(6,12,15,16)$.

Specific stimulation of Fas antigen can easily induce apoptotic cell death in differentiated human endometrial adenocarcinoma HHUA cells (17), and Fas-mediated apoptosis can be enhanced in a dose-dependent manner by targeted knockdown of endogenous DAPK protein expression by treatment with specific DAPK siRNAs (DAPK-KD) (18). Since DAPK-KD induces tumor necrosis factor-related apoptosis-inducing ligand (TRAIL)-mediated apoptosis in HHUA cells (15), it is highly possible that DAPK-mediated signals constitutively suppress the tumor necrosis factor receptor-mediated cell death signaling common to the Fas-mediated and TRAIL-mediated pathways. Although typical apoptosis with DNA fragmentation can easily be induced in HHUA cells by treatment with 5-fluorouracil (5FU) (19), DAPK-KD increases apoptotic susceptibility to 5FU in HHUA cells (20). However, DAPK-KD has no effect on etoposide (VP16)-sensitivity in HHUA cells (20). Therefore, there are two apoptotic signals in HHUA cells, comprising DAPK-regulated and DAPK-independent death signals.

In the human cervical squamous cell carcinoma cell line ME180, which exhibits hypermethylation of normally unmethylated CpG islands in the promoter region of the DAPK gene (13), DAPK protein expression is constitutively suppressed but can be strongly induced by treatment with the demethylating agent 5-aza-2'-deoxycytidine (5-aza-CdR) (21). However, in ME180derived cisplatin (CDDP)-resistant cell lines, DAPK protein expression cannot be induced by treatment with 5 -aza-CdR. 
Since DAPK mRNA is expressed in the CDDP-resistant cells in a similar manner to the parent ME180 cells, the strong suppression of DAPK protein induction after 5-aza-CdR treatment may be a result or an indicator of acquired CDDP-resistance (21). On the other hand, DAPK-KD enhances 5FU-sensitivity but not VP16-sensitivity in HHUA cells (20), in which DAPK protein is highly expressed (13). These observations suggest that DAPK protein expression levels can regulate cellular sensitivity to certain anticancer drugs (20).

Recent anticancer chemotherapies have improved the overall survival rates of advanced cancer patients. 5FU has often been administered to advanced gastric and colorectal cancer patients for more than 20 years, and a recent combination chemotherapy with 5FU and oxaliplatin has brought about a remarkable improvement in the long-term survival rates of inoperable colorectal cancer patients (22). Consequently, this combination chemotherapy with $5 \mathrm{FU}$ and oxaliplatin has also been applied to patients with recurrent ovarian cancer after standard chemotherapy with paclitaxel and carboplatin $(23,24)$. Since $5 F U$ combination chemotherapies have been reapplied to various cancer patients in addition to colorectal cancer patients, 5FU-resistance may become a large clinical problem in the current cancer chemotherapies. However, there are few recent basic studies regarding the mechanisms of acquired 5FU-resistance and cross-resistance to 5FU and other new anticancer drugs. Investigations of the mechanisms of 5FU-resistance may lead to the development of novel effective anticancer chemotherapies for 5FU-resistant patients.

To clarify the molecular mechanisms of acquired 5FU-resistance in cancer cells, we previously established several monoclonal 5FU-resistant subclones from HHUA cells (19). DAPK constitutively suppresses 5FU-stimulated cell death in the parent HHUA cells, while all the 5FU-resistant subclones established from HHUA cells show not only 5FU-resistance but also crossresistance to various anticancer drugs (19). The 5FU-resistant subclones also show significantly reduced sensitivity to Fas-mediated apoptosis. In this study, we investigated the changes in the DAPK-mediated survival signals against 5FU-stimulated apoptosis using the multidrug-resistant 5FU-resistant HHUA subclones to analyze the mechanisms of the multidrug-resistance.

\section{Materials and methods}

Cell line and culture. The HHUA cell line (25) was obtained from the Riken Cell Bank (Tsukuba, Japan). The cells were cultured in Opti-MEM (Invitrogen Corp., Carlsbad, CA) supplemented with 5\% fetal bovine serum (Equitech Bio Inc., Ingram, TX), penicillin $(100 \mathrm{U} / \mathrm{ml})$, streptomycin $(100 \mathrm{U} / \mathrm{ml})$ and Fungizone $\left(0.25 \mu \mathrm{g} / \mathrm{ml}\right.$; Invitrogen Corp.) under $5 \% \mathrm{CO}_{2}$ and $95 \%$ air at $37^{\circ} \mathrm{C}$.

Transfection of DAPK siRNAs. Two DAPK siRNA duplexes, DAPK siRNA1 and siRNA2, were designed and synthesized by iGENE Therapeutics Inc. (Tsukuba, Japan). The two siRNA sequences are shown in Table I. A negative control siRNA was purchased from Ambion Inc. (Austin, TX). Lipofectamine 2000 (Invitrogen Corp.) was used as the transfection reagent according to the manufacturer's instructions. For experiments, cells were seeded in 6 -well plates $\left(2.5 \times 10^{5}\right.$ cells/well $)$ or $10-\mathrm{cm}$ dishes ( $2 \times 10^{6}$ cells/dish), cultured for $24 \mathrm{~h}$ and then transfected with the DAPK siRNAs or control siRNA at a final concentration of $50 \mathrm{nM}$. Subsequently, the cells were cultured for $24-48 \mathrm{~h}$ for mRNA analyses and 48-72 $\mathrm{h}$ for protein assays before being harvested as indicated.

Cell viability assay. A Cell Counting kit (Dojindo Chemical Laboratory Co. Ltd., Tokyo, Japan) was used to evaluate the abilities of the DAPK siRNAs to enhance the cytotoxic effects of 5FU (provided by Kirin-Kyowa-Hakko Co. Ltd., Tokyo, Japan). Cells were plated in 6-wells of 96-well plates at $5 \times 10^{3}$ cells/well and cotransfected with Lipofectamine 2000 and 50 nM DAPK siRNAs or control siRNA. At $24 \mathrm{~h}$ after transfection, the cells were incubated with various concentrations of $5 \mathrm{FU}$ for a further 48-72 h. At the end of the treatments, the viable cell numbers were determined using the Cell Counting kit according to the manufacturer's instructions. The absorbances at $450 \mathrm{~nm}$ were measured using a microplate reader. Cell viability of $100 \%$ was defined as the absorbance obtained for cells without 5FU treatment. All experiments were performed in triplicate. Data are expressed as the mean $\pm S D$, and comparative data $(n=6)$ were analyzed by ANOVA. The level of statistical significance was set at $\mathrm{p}<0.05$.

DAPK-methylation-specific PCR (DAPK-MS-PCR). Genomic DNA was isolated from cultured cells using a SepaGene kit (Sanko-Junyaku Ltd., Tokyo, Japan) according to the manufacturer's instructions. The DNA concentrations were calculated from the UV absorptions at 260 and $280 \mathrm{~nm}$. DAPKMS-PCR was performed as described previously (21). Briefly, a bisulfite-modified DNA was used as a template for stage I PCR amplification to generate a 209-bp fragment of the DAPK gene that included a portion of its $\mathrm{CpG}$-rich promoter region. The stage I PCR primers used recognized the modified DNA but did not discriminate between methylated and unmethylated alleles. The stage I PCR amplification was carried out as follows: $95^{\circ} \mathrm{C}$ for $15 \mathrm{~min}$; 35 cycles of denaturation at $94^{\circ} \mathrm{C}$ for $1 \mathrm{~min}$, annealing at $58^{\circ} \mathrm{C}$ for $150 \mathrm{sec}$ and extension at $72^{\circ} \mathrm{C}$ for $150 \mathrm{sec}$; and a final extension at $72^{\circ} \mathrm{C}$ for $10 \mathrm{~min}$. The stage I PCR products were diluted by 1:50, and $5 \mu \mathrm{l}$ aliquots were subjected to stage II PCR amplification using primers specifically designed for methylated or unmethylated DNA in the promoter region of the DAPK gene. The primers used for the stage I and II PCR amplifications were described previously (21). For the stage II PCR amplification, the annealing temperature was increased to $65^{\circ} \mathrm{C}$ and the annealing time was reduced to $90 \mathrm{sec}$ for 40 cycles. The stage II PCR amplified 153- and 106-bp products from the methylated and unmethylated DAPK genes, respectively. Finally, the PCR products were electrophoresed in a $2.0 \%$ agarose gel at $100 \mathrm{~V}$ for $\sim 30-40 \mathrm{~min}$, and visualized by staining with $5 \mu \mathrm{g} / \mathrm{ml}$ ethidium bromide.

RT-PCR of the DAPK gene. Total RNA was isolated from cultured cells using the TRIzol reagent (Invitrogen Corp.) for RT-PCR analyses or an RNeasy Mini Kit (Qiagen Inc., Valencia, CA) for real-time RT-PCR analyses. Aliquots containing $1 \mu \mathrm{g}$ of total RNA were pretreated with DNase I (Invitrogen Corp.) and then subjected to cDNA synthesis using a reverse transcriptase kit (Bio-Rad, Hercules, CA) in a reaction volume of $20 \mu \mathrm{l}$. Each cDNA product was diluted to $100 \mu \mathrm{l}$. The PCR mixture $(25 \mu \mathrm{l})$ contained $5 \mu \mathrm{l}$ of diluted cDNA, $0.125 \mu \mathrm{l}$ of Hotstart polymerase (Qiagen Inc.), $0.2 \mathrm{mM}$ dNTP, $1 \mathrm{xQ}$ solution and $0.5 \mu \mathrm{M}$ primers. 
Table I. DAPK siRNA sequences.

\begin{tabular}{ll}
\hline DAPK siRNA & siRNA sequence \\
\hline DAPK siRNA-1 & 5'-CAACAUCAUGCAAAGUGAAACAGUU-AG-3' \\
& 3'-AU-GUUGUAGUACGUUUCACUUUGUCAA-5' \\
DAPK siRNA-2 & 5'-AGCCAAGAAUUAAGCUCAAGCUGUU-AG-3' \\
& 3'-AU-UCGGUUCUUAAUUCGAGUUCGACAA-5'
\end{tabular}

The primers used for DAPK were described in a previous report (21). $\beta$-actin was evaluated as a positive control for the mRNA amount. For RT-PCR analyses, an initial hot start at $95^{\circ} \mathrm{C}$ for $15 \mathrm{~min}$ was followed by 35 amplification cycles of $30 \mathrm{sec}$ at $94^{\circ} \mathrm{C}, 30 \mathrm{sec}$ at the annealing temperature and $60 \mathrm{sec}$ at $72^{\circ} \mathrm{C}$. The annealing temperature was $55^{\circ} \mathrm{C}$ for DAPK. The PCR products were electrophoresed in 1.5-2.0\% agarose gels at 100 $\mathrm{V}$ for $\sim 30-40 \mathrm{~min}$ and visualized by staining with $5 \mu \mathrm{g} / \mathrm{ml}$ ethidium bromide.

Western blot analysis of DAPK protein expression. The HHUA parent cells and 5FU-resistant subclones were harvested and lysed with $0.3 \mathrm{ml}$ of lysis buffer (Sigma, St. Louis, MO). The protein contents of the cell lysates were quantified by a Coomassie Plus Protein Assay (Pierce Biotechnology Inc., Rockford, IL) and aliquots (25 $\mu \mathrm{g}$ total protein) were dissolved in Laemmli SDS-PAGE sample buffer prior to separation by 7.5\% SDS-PAGE. The separated proteins were transferred to a polyvinylidene fluoride membrane (ATTO Corp., Tokyo, Japan) using a wet transfer method. The membrane was blocked with $5 \%$ skim-milk for $1 \mathrm{~h}$ at room temperature and subsequently incubated with a mouse monoclonal anti-human DAPK antibody (clone 55; 1:5000 dilution; Sigma) for $1 \mathrm{~h}$ at room temperature. After washing with TBS-T (20 mM Tris-HCl pH 7.6, 0.3 M NaCl, $0.1 \%$ Tween-20), the membrane was incubated with a horseradish peroxidase-conjugated rabbit anti-mouse secondary antibody (Sigma) for $1 \mathrm{~h}$ at room temperature. The bound antibodies were detected with an ECL Plus kit (Amersham Pharmacia Biotech, Uppsala, Sweden) and the membrane was scanned using a chemiluminescence imaging system (Luminocapture AE6955; ATTO Corp.).

Semiquantitative flow cytometric analysis of cell surface antigens. Cells were detached from culture flasks with $3 \mathrm{mM}$ EDTA in phosphate-buffered saline (PBS), and immunostained as follows. The cells $\left(3 \times 10^{5}\right)$ were incubated with an excess of the primary antibody for $20 \mathrm{~min}$ at $4^{\circ} \mathrm{C}$ and then washed twice with wash buffer (PBS containing $2 \%$ fetal calf serum and $0.1 \% \mathrm{NaN}_{3}$ ). Next, the cells were incubated with FITCconjugated goat anti-mouse IgG $(\mathrm{H}+\mathrm{L})$ (Dako Japan, Kyoto, Japan) for $20 \mathrm{~min}$ at $4^{\circ} \mathrm{C}$ and washed twice with wash buffer. Finally, the cells were suspended in $200 \mu \mathrm{l}$ of wash buffer and analyzed using a FACSCalibur ${ }^{\mathrm{TM}}$ (Beckman Coulter Japan, Tokyo, Japan). The following primary antibodies were used: mouse anti-human CD29 monoclonal antibody (clone K20; Immunotech, Hampshire, UK); mouse anti-human CD49a monoclonal antibody (clone TS2/7; Serotec Ltd., Oxford, UK); mouse anti-human CD49b monoclonal antibody (clone 31H4;
Serotec Ltd.); mouse anti-human CD49c monoclonal antibody (clone 11G5; Cymbus Biotech Ltd.); mouse anti-human CD49d monoclonal antibody (clone 44H6; Cymbus Biotech Ltd.); mouse anti-human CD49e monoclonal antibody (clone SAM1; Beckman Coulter Japan); mouse anti-human CD49f monoclonal antibody (clone 4F10; Cymbus Biotech Ltd.); mouse anti-human CD40 monoclonal antibody (clone mAb89; Immunotech); and mouse anti-human Fas (CD95) monoclonal IgG (clone UB2; MBL, Nagoya, Japan).

Semiquantitative flow cytometric analysis of intracellular molecules. Flow cytometric analyses of intracellular molecules were performed as follows. Cells were detached from culture flasks with $3 \mathrm{mM}$ EDTA in PBS, and washed with wash buffer (PBS containing $2 \%$ fetal calf serum). The cells were fixed with $4 \%$ paraformaldehyde in $0.1 \mathrm{M} \mathrm{NaH}_{2} \mathrm{PO}_{4}(\mathrm{pH} 7.4)$ for $10 \mathrm{~min}$ on ice. After two washes with wash buffer, the cells were treated with $100 \mathrm{mg} / \mathrm{ml}$ digitonin (Sigma) for $10 \mathrm{~min}$ at room temperature. After another two washes, the cells were treated with normal mouse IgG to block non-specific binding sites for $5 \mathrm{~min}$ at room temperature, followed by incubation with the primary antibody for $30 \mathrm{~min}$ at room temperature and two further washes. Finally, the cells were incubated in FITC-conjugated goat anti-mouse IgG (Gibco BRL, Carlsbad, CA) for $30 \mathrm{~min}$ at room temperature, washed twice and suspended in $200 \mu \mathrm{l}$ of wash buffer for analyses using a FACSCalibur (Beckman Coulter Japan). The following primary antibodies were used: mouse anti-human p53 monoclonal antibody (clone MAb421; Enzo Life Sci, Plymouth Meeting, PA); mouse anti-human BCL-2 monoclonal antibody (clone 100; AbD Serotec, Oxford, UK); mouse anti-human BAX monoclonal antibody (clone 2D2; AbD Serotec); mouse anti-human BCL-X monoclonal antibody (clone 2H12; Acris Antibodies, San Diego, CA); mouse anti-human lung-resistance protein (LRP) monoclonal antibody (clone LRP-56; Merck Millipore, Darmstadt, Germany); and mouse anti-human DAPK monoclonal antibody.

\section{Results}

Effects of DAPK-KD on the 5FU-resistant subclones. Using three monoclonal 5FU-resistant subclones derived from HHUA cells (5FUr3C, 5FU3D and 5FUr4A) that show multidrugresistance (19), the effects of DAPK-KD on the 5FU-sensitivity of the subclones were analyzed. A previous report demonstrated that transfection of the two DAPK siRNAs (DAPK siRNA1 and siRNA2) used in the study strongly and specifically suppresses DAPK protein expression in HHUA cells (17). As shown in Fig. 1, DAPK-KD with either DAPK siRNA1 or DAPK 

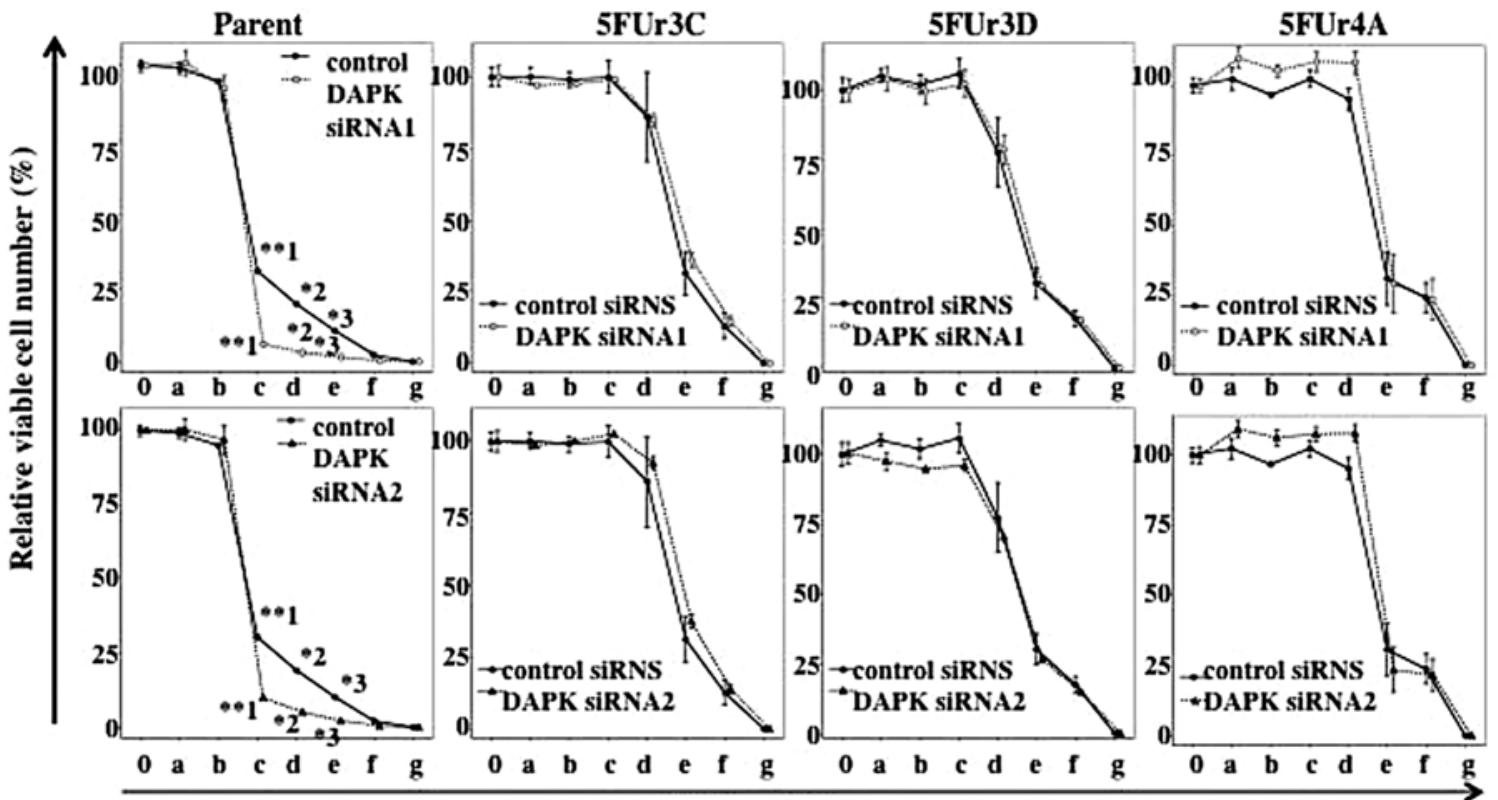

$5 \mathrm{FU}(\mu \mathrm{g} / \mathrm{ml})$

Figure 1. Effects of DAPK-KD on the 5FU-sensitivity of HHUA cells. The 5FU-sensitivities of the parent cells and three 5FU-resistant subclones were examined The solid lines with closed circles show control siRNA-transfected cells. The dotted lines with open circles show DAPK siRNA-transfected cells. The effects of transfection of $50 \mathrm{nM}$ DAPK siRNA1 (upper graphs) or siRNA2 (lower graphs) on the 5FU dose-dependent decrease in cell viability were assayed. The effects of the control siRNA were simultaneously assayed at a concentration of $50 \mathrm{nM}$. Transfection of each DAPK siRNA dose-dependently enhances 5FU-mediated cell death in the parent cells but not in the three 5FU-resistant subclones. The final concentrations $(\mathrm{mg} / \mathrm{ml})$ of $5 \mathrm{FU}$ indicated as 0 and a-g at the bottom of the figures were as follows: $0,0.32,1.6,8,40,200,1000$ and $5000 \mathrm{mg} / \mathrm{ml}^{* *} 1, \mathrm{p}<0.01 ;{ }^{*} 2$ and ${ }^{*} 3, \mathrm{p}<0.05$.

siRNA2 significantly enhanced the 5FU-sensitivity of the parent HHUA cells but did not affect the 5FU-sensitivities of the three 5FU-resistant subclones.

Regulation of DAPK expression in the 5FU-resistant subclones. To investigate the mechanisms of the different 5FU-sensitivities after DAPK-KD between the parent cells and 5FU-resistant cells, we examined the DAPK expression in the 5FU-resistant subclones. Although epigenetic downregulation of DAPK gene expression by hypermethylation of its promoter region has been reported in various cancer cells (13), no hypermethylation of the DAPK gene was observed in the parent cells or 5FU-resistant cells (Fig. 2A). Moreover, RT-PCR analyses showed that there were no apparent differences in the DAPK mRNA expression patterns between the parent cells and 5FU-resistant subclones (Fig. 2B), although it was reported that there is only a lack of relationship between the hypermethylation status of the DAPK promoter and the DAPK mRNA expression levels in drugresistant subclones derived from ME180 cells $(21,26)$. Although DAPK protein expression is strongly suppressed in CDDPresistant ME180 cells with high DAPK mRNA expression levels (21), the 5FU-resistant cells expressed DAPK protein at similar levels to the parent cells (Fig. 2C), suggesting that there was no impairment of DAPK mRNA translation in the 5FU-resistant HHUA cells, unlike the case for CDDP-resistant ME180 cells.

Semiquantitative flow cytometric analysis of cell surface antigen expressions on the $5 F U$-resistant subclones. In the 5FU-resistant cells, the 5FU-sensitivity was not enhanced by DAPK-KD and there were no apparent differences in the DAPK promoter methylation and DAPK protein expression compared with the parent cells. Therefore, we carried out semiquantitative flow cytometric
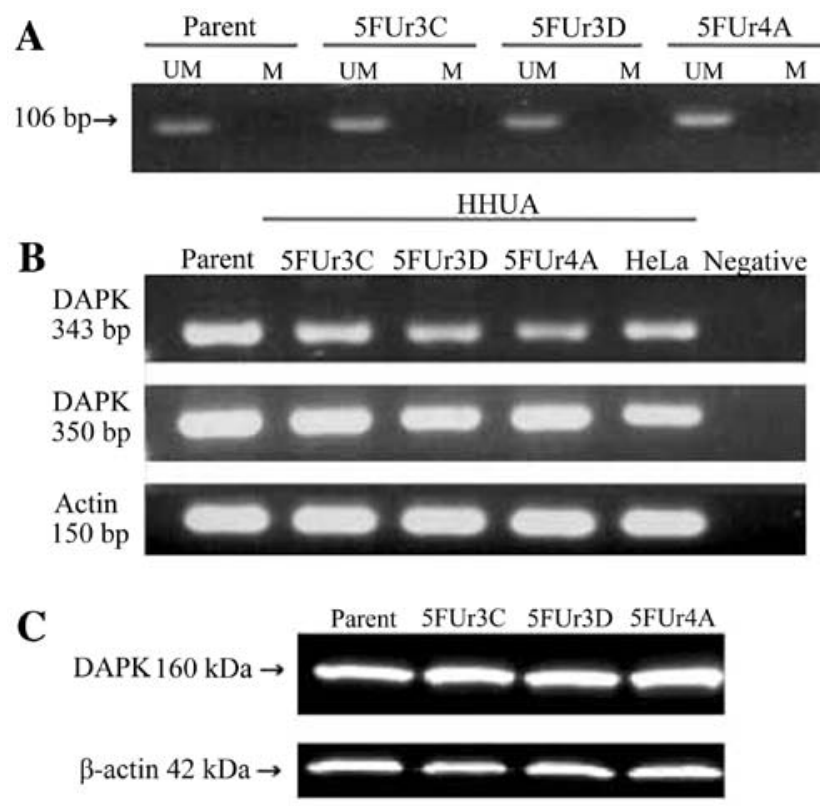

Figure 2.DAPK expressions in the 5FU-resistant subclones.(A) MS-DAPK-PCR analyses of the 5FU-resistant subclones. Methylation-specific PCR of the DAPK gene was performed in the HHUA parent cells and 5FU-resistant subclones. The parent cells and all three 5FU-resistant subclones only show unmethylated DAPK bands. U, unmethylated; M, methylated. (B) RT-PCR analyses of DAPK mRNA expressions in the 5FU-resistant subclones. All three 5FU-resistant subclones express DAPK mRNA at similar levels to the parent cells. (C) DAPK protein expressions in the parent cells and 5FU-resistant subclones. All three 5FU-resistant subclones express DAPK protein at similar levels to the parent cells.

analyses of the expression levels of cell surface antigens that are known to regulate cell survival or cell death. As shown in Fig. 3, 


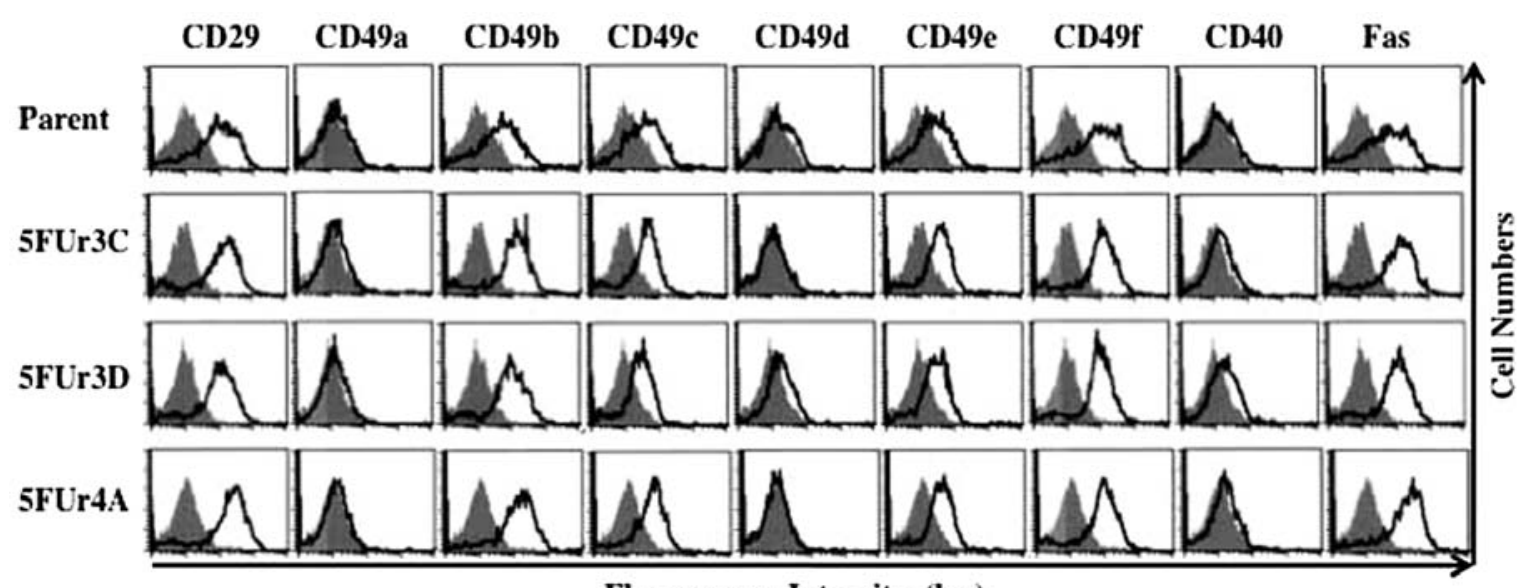

Fluorescence Intensity (log)

Figure 3. Semiquantitative flow cytometric analyses of the cell surface antigen expressions on the 5FU-resistant subclones. In the panels, the dark gray histograms show the negative controls and the white histograms show the positive reactions.

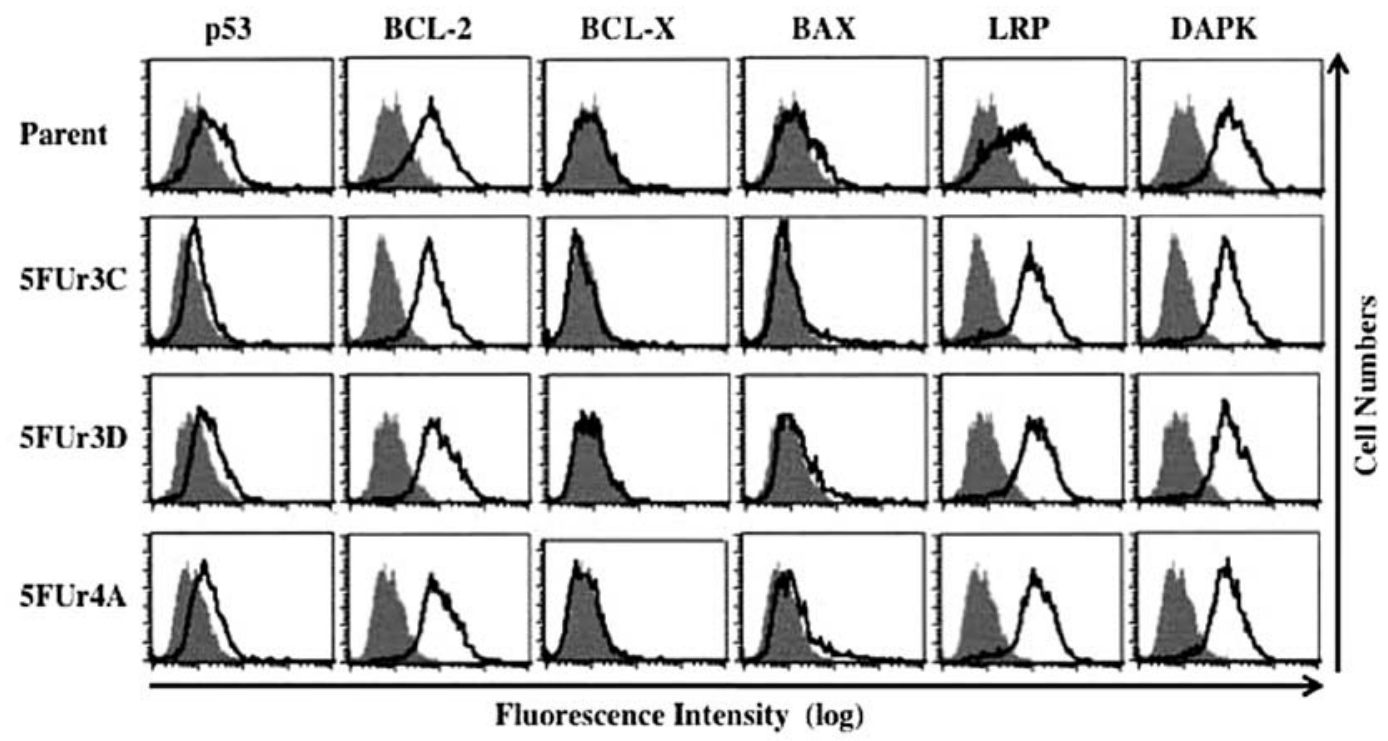

Figure 4. Semiquantitative flow cytometric analyses of the intracellular apoptosis-regulating molecule expressions in the 5FU-resistant subclones. In the panels, the dark gray histograms show the negative controls and the white histograms show the positive reactions.

there were no apparent differences in the expression levels of the nine surface antigens examined between the parent cells and 5FU-resistant cells.

Semiquantitative flow cytometric analysis of intracellular apoptosis-regulating molecule expressions in the $5 F U$-resistant subclones. Although susceptibility to Fas-mediated apoptosis is reduced in the 5FU-resistant cells (18), there were no differences in the cell surface Fas antigen expression levels between the parent cells and 5FU-resistant cells (Fig. 3). These findings indicate that the reduced sensitivity to Fas-mediated apoptosis in the 5FU-resistant cells may be caused by impairment of intracellular cell death signals after Fas stimulation. Therefore, semiquantitative flow cytometric analyses were performed to evaluate the protein expression levels of intracellular apoptosisregulating molecules in the 5FU-resistant subclones. As shown in Fig. 4, there were no apparent differences in the DAPK protein expression levels between the parent cells and 5FU-resistant cells, similar to the case for the western blot analyses (Fig. 2C). Regarding the other apoptosis-regulating molecules examined, namely p53, BCL-2, BAX, BCL-X and LRP, the 5FU-resistant subclones showed no differences in their expression levels compared with the parent cells.

\section{Discussion}

This study may be the first to show that DAPK-mediated survival signals are impaired in 5FU-resistant cancer cells. Moreover, the mechanisms of multidrug-resistance associated with 5FU-resistance acquisition were also investigated.

In a previous study, DAPK-KD in five human uterine cancer cell lines, including HHUA cells, was found to specifically induce apoptotic cell death in all five cell lines (16). These findings suggest that DAPK plays essential survival roles in the cells and may be a candidate for anticancer molecular targeting therapies in certain human uterine cancer cells. However, DAPK does 
not have a survival function in the human cervical squamous cancer cell line ME180, in which DAPK protein expression is strongly suppressed and anticancer drug-sensitivities are not correlated with DAPK protein expression levels $(21,26)$. These observations indicate that the apoptotic or anti-apoptotic functions of DAPK may be dependent on the cell lineage or that ME180 cells may be cancer cells lacking DAPK-mediated survival signals. DAPK-KD enhances Fas-mediated apoptosis (18) and 5FU-stimulated apoptosis (20) in HHUA parent cells, but does not affect VP16-stimulated cell death in HHUA cells (20). Therefore, the DAPK-mediated survival signals in HHUA cells suppress the apoptotic signals mediated by either Fas or 5FU stimulation, but are independent of VP16-stimulated apoptotic signals. Since the 5FU-resistant HHUA subclones show multidrug-resistance (19), analyses of the DAPK-mediated survival signals in the 5FU-resistant subclones can partly clarify the mechanisms of acquired multidrug-resistance.

Unlike the parent HHUA cells, DAPK-KD did not enhance the 5FU-sensitivities and susceptibilities to Fas-stimulated apoptosis or cause any significant changes of the cell surface Fas antigen expression levels in the 5FU-resistant subclones, indicating the possibility that the DAPK-mediated common antiapoptotic/survival signaling pathways between Fas-mediated apoptosis and 5FU-stimulated apoptosis were impaired in the 5FU-resistant cells. However, the 5FU-resistant cells have also acquired resistance to VP16-stimulated apoptosis, a DAPKindependent apoptosis (20).

Based on these findings, we postulate the following hypothesis. In the parent HHUA cells, DAPK is an essential survival molecule which suppresses Fas-mediated apoptosis and 5FU-stimulated apoptosis, while the 5FU-resistant subclones are not only insensitive to both Fas-mediated apoptosis and 5FU-stimulated apoptosis but also resistant to DAPK-independent VP16-stimulated apoptosis. In fact, the 5FU-resistant subclones have acquired strong multidrug-resistance against CDDP, SN38 (an active metabolite of irinotecan), 4-OH-cyclophosphamide (an active metabolite of cyclophosphamide) and paclitaxel, in addition to 5FU and VP16 (19). Therefore, the possible mutated sites in the 5FU-resistant cells may have common apoptotic signals related to Fas-mediated apoptosis and the cell deaths stimulated by various anticancer drugs. Otherwise, the 5FU-resistant cells may have multiple mutations in the various apoptotic signaling pathways. The 5FU-resistant cells have acquired a stronger survival activity through impaired apoptotic signals common to various apoptotic stimuli. ME180 cells, in which DAPK does not have any survival activity, may have constitutively impaired apoptotic signals, similar to the case for the 5FU-resistant HHUA cells, and show strong survival activity.

Identification of the mutated sites in the 5FU-resistant cells might lead to efficient methods to restore the sensitivities to anticancer drugs in multidrug-resistant cancer cells. The 5FU-resistant subclones show a normal karyotype like the parent HHUA cells (19), and our semiquantitative flow cytometric analyses did not find any differences in nine cell surface antigens and six intracellular molecules that may regulate various cell survival and cell death processes. These findings suggest that the 5FU-resistant subclones may have a few gene mutations or small chromosome changes. We are now investigating the mutated sites in the 5FU-resistant cells.

\section{References}

1. Deiss LP, Feinstein E, Berissi H, Cohen O and Kimchi A: Identification of a novel serine/threonine kinase and a novel $15-\mathrm{kDa}$ protein as potential mediators of the gamma interferoninduced cell death. Genes Dev 9: 15-30, 1995.

2. Cohen O, Feinstein E and Kimchi A: DAP kinase is a $\mathrm{Ca}^{2+} /$ calmodulin-dependent, cytoskeleton-associated protein kinase, with cell death-inducing functions that depend on its catalytic activity. EMBO J 16: 998-1008, 1997.

3. Kissil JL, Feinstein E, Cohen O, Jones PA, Tsai YC, Knowles MA, Eydmann ME and Kimchi A: DAP kinase loss of expression in various carcinoma and B-cell lymphoma cell lines: possible implications for role as tumor suppressor gene. Oncogene 15: 403-407, 1997.

4. Inbal B, Cohen O, Polak-Charcon S, Kopolovic J, Vadai E, Eisenbach L and Kimchi A: DAP kinase links the control of apoptosis to metastasis. Nature 390: 180-184, 1997.

5. Cohen O, Inbal B, Kissil JL, Raveh T, Berissi H, SpivakKroizaman T, Feinstein E and Kimchi A: DAP kinase participates in TNF-alpha- and Fas-induced apoptosis and its function requires the death domain. J Cell Biol 146: 141-148, 1999.

6. Jang CW, Chen CH, Chen CC, Chen JY, Su YH and Chen RH: TGF $\beta$ induces apoptosis through Smad-mediated expression of DAP kinase. Nat Cell Biol 4: 51-58, 2002.

7. Raveh T, Droguett G, Horwitz MS, De Pinho RA and Kimchi A: DAP kinase activates a p19ARF/p53-mediated apoptotic checkpoint to suppress oncogenic transformation. Nat Cell Biol 3: 1-7, 2001.

8. Pelled D, Raveh T, Riebeling C, Fridkin M, Berissi H, Futerman AH and Kimchi A: Death-associated protein (DAP) kinase plays a central role in ceramide induced apoptosis in cultured hippocampal neurons. J Biol Chem 277: 1957-1961, 2002.

9. Yamamoto M, Hioki T, Nakajima-Iijima S and Uchino S: DAP kinase activity is critical for C (2)-ceramide-induced apoptosis in PC12 cells. Eur J Biochem 269: 139-147, 2002.

10. Wang WJ, Kuo JC, Yao CC and Chen RH: DAP kinase induces apoptosis by suppressing integrin activity and disrupting matrix survival signals. J Cell Biol 159: 169-179, 2002.

11. Jin Y, Blue EK, Dixon S, Hou L, Wysolmerski RB and Gallagher PJ: Identification of a new form of death-associated protein kinase that promotes cell survival. J Biol Chem 276: 39667-39678, 2001.

12. Jin Y and Gallagher PJ: Antisense depletion of death-associated protein kinase promotes apoptosis. J Biol Chem 278: 51587-51593, 2003.

13. Bai T, Tanaka T, Yukawa K and Umesaki N: Reduced expression of death-associated protein kinase in human uterine and ovarian carcinoma cells. Oncol Rep 11: 661-665, 2003.

14. Li L, Tanaka T, Yukawa K, Akira S and Umesaki N: Irinotecanindiced ovarian follicular apoptosis is atteuated by deleting the kinase domain of death-associated protein kinase. Int J Oncol 34: 905-914, 2009.

15. Bai T, Tanaka T and Yukawa K: Targeted knockdown of deathassociated protein kinase expression induces TRAIL-mediated apoptosis in human endometrial adenocarcinoma cells. Int $\mathbf{J}$ Oncol 37: 203-210, 2010.

16. Tanaka T, Bai T and Yukawa K: Death-associated protein kinase is essential for the survival of various types of uterine cancer cells. Int J Oncol 37: 1017-1022, 2010.

17. Tanaka $\mathrm{T}$ and Umesaki N: Cytokine regulation of apoptotic susceptibility in a human endometrial epithelial cell line. J Reprod Immunol 47: 105-119, 2000.

18. Tanaka T, Bai T and Yukawa K: Specific downregulation of deathassociated protein kinase enhances Fas-mediated apoptosis in the human differentiated endometrial adenocarcinoma cell line, HHUA. Eur J Gynaecol Oncol 32: 293-296, 2011.

19. Tanaka T, Bai T and Toujima S: Establishment and characterization of monoclonal 5-fluorouracil-resistant cell lines derived from human endometrial adenocarcinoma. Int J Oncol 37: 731-736, 2010.

20. Tanaka T, Bai T and Yukawa K: Suppressed protein expression of death-associated protein kinase enhances 5 -fluorouracil-sensitivity but not etoposide-sensitivity in human endometrial adenocarcinoma cells. Oncol Rep 24: 1401-1405, 2010.

21. Bai T, Tanaka T, Yukawa K and Umesaki N: A novel mechanism for acquired cisplatin-resistance: Suppressed translation of death-associated protein kinase mRNA is insensitive to 5-aza-2'-deoxycitidine and trichostatin in cisplatin-resistant cervical squamous cancer cells. Int J Oncol 28: 497-508, 2006. 
22. de Gramont A, Figer A, Seymour M, Homerin M, Hmissi A, Cassidy J, Boni C, Cortes-Funes H, Cervantes A, Freyer G, Papamichael D, Le Bail N, Louvet C, Hendler D, de Braud F, Wilson C, Morvan F and Bonetti A: Leucovorin and fluorouracil with or without oxaliplatin as first-line treatment in advanced colorectal cancer. J Clin Oncol 18: 2938-2947, 2000.

23. Pectasides D, Pectasides M,Farmakis D, Gaglia A, Koumarianou A, Nikolaou M, Koumpou M, Kountourakis P,Papaxoinis G, Mitrou P Economopoulos T and Raptis SA: Oxaliplatin plus high-dose leucovorin and 5-fluorouracil (FOLFOX 4) in platinum-resistant and taxane-pretreated ovarian cancer: a phase II study. Gynecol Oncol 95: 165-172, 2004.

24. Rosa DD, Awada A, Mano MS, Selleslags J, Lebrun F, Gil T, Piccart MJ and D'Hondt V: Oxaliplatin/5fluorouracil-based chemotherapy was active and well tolerated in heavily pretreated patients with ovarian carcinoma. Arch Gynecol Obstet 278: 457-462, 2008.
25. Ishiwata I, Ishiwata $\mathrm{C}$, Soma $\mathrm{M}$, Arai J and Ishikawa $\mathrm{H}$ : Establish-ment of human endometrial adenocarcinoma cell line containing estradiol-17 beta and progesteron receptors. Gynecol Oncol 17: 281-290, 1984.

26 Tanaka T, Bai T, Toujima S, Utsunomiya T, Matsuoka T, Kobayashi A, Yamamoto M, Sasaki N, Tanizaki Y, Utsunomiya H, Tanaka J and Yukawa K: Demethylation restores SN38-sensitivity in acquired SN38-resistant cells derived from human cervical squamous cancer cells. Oncol Rep 27: 1292-1298, 2012 . 